\title{
Essential Elements of Effective Workplace Programs and Policies for Improving Worker Health and Wellbeing
}

\section{Introduction}

The Essential Elements of Effective Workplace Programs and Policies for Improving Worker Health and Wellbeing is a resource document developed by the National Institute for Occupational Safety and Health (NIOSH) with substantial input from experts and interested individuals.

This document, a key part of the NIOSH WorkLife Initiative, is intended as a guide for employers and employeremployee partnerships wishing to establish effective workplace programs that sustain and improve worker health. The Essential Elements document identifies twenty components of a comprehensive work-based health protection and health promotion program and includes both guiding principles and practical direction for organizations seeking to develop effective workplace programs.

The WorkLife Initiative is intended to identify and support comprehensive approaches to reduce workplace hazards and promote worker health and well being. The premise of this Initiative, based on scientific research and practical experience in the field, is that comprehensive practices and policies that take into account the work environment--both physical and organizational-- while also addressing the personal health risks of individuals, are more effective in preventing disease and promoting health and safety than each approach taken separately.

The twenty components of the Essential Elements, presented below, are divided into four areas: Organizational Culture and Leadership; Program Design; Program Implementation and Resources; and Program Evaluation. The document is a framework that will be enhanced by links to resource materials intended to assist in the design and implementation of workplace programs and offer specific examples of best and promising practices.

\section{Organizational Culture and Leadership}

1. Develop a "Human Centered Culture." Effective programs thrive in organizations with policies and programs that promote respect throughout the organization and encourage active worker participation, input, and involvement. A Human Centered Culture is built on trust, not fear.

2. Demonstrate leadership. Commitment to worker health and safety, reflected in words and actions, is critical. The connection of workforce health and safety to the core products, services and values of the company should be acknowledged by leaders and communicated widely. In some notable examples, corporate Boards of Directors have recognized the value of workforce health and wellbeing by incorporating it into an organization's business plan and making it a key operating principle for which organization leaders are held accountable.

3. Engage mid-level management. Supervisors and managers at all levels should be involved in promoting health-supportive programs. They are the direct links between the workers and upper management and will determine if the program succeeds or fails. 
Mid level supervisors are the key to integrating, motivating and communicating with

employees.

\section{Program Design}

4. Establish clear principles. Effective programs have clear principles to focus priorities, guide program design, and direct resource allocation. Prevention of disease and injury supports worker health and well being.

5. Integrate relevant systems. Program design involves an initial inventory and evaluation of existing programs and policies relevant to health and well-being and a determination of their potential connections. In general, better integrated systems perform more effectively. Programs should reflect a comprehensive view of health: behavioral health/mental health/physical health are all part of total health. No single vendor or provider offers programs that fully address all of these dimensions of health. Integrate separately managed programs into a comprehensive health-focused system and coordinate them with an overall health and safety management system. Integration of diverse data systems can be particularly important and challenging.

6. Eliminate recognized occupational hazards. Changes in the work environment (such as reduction in toxic exposures or improvement in work station design and flexibility) benefit all workers. Eliminating recognized hazards in the workplace is foundational to WorkLife principles.

7. Be consistent. Workers' willingness to engage in worksite health-directed programs may depend on perceptions of whether the work environment is truly health supportive. Individual interventions can be linked to specific work experience. Change the physical and organizational work environment to align with health goals. For example, blue collar workers who smoke are more likely to quit and stay quit after a worksite tobacco cessation program if workplace dusts, fumes, and vapors are controlled and workplace smoking policies are in place.

8. Promote employee participation. Ensure that employees are not just recipients of services but are engaged actively to identify relevant health and safety issues and contribute to program design and implementation. Barriers are often best overcome through involving the participants in coming up with solutions. Participation in the development, implementation, and evaluation of programs is usually the most effective strategy for changing culture, behavior, and systems.

9. Tailor programs to the specific workplace and the diverse needs of workers. Workplaces vary in size, sector, product, design, location, health and safety experience, resources, and worker characteristics such as age, training, physical and mental abilities, resiliency, education, cultural background, and health practices. Successful programs recognize this diversity and are designed to meet the needs of both individuals and the enterprise. Effective programs are responsive and attractive to a diverse workforce. One size does not fit all-flexibility is necessary.

10. Consider incentives and rewards. Incentives and rewards, such as financial rewards, time off, and recognition, for individual program participation may encourage engagement, although poorly designed incentives may create a sense of "winners" and "losers" and have unintended adverse consequences. Vendors' contracts should have incentives and rewards aligned with accomplishment of program objectives.

11. Find and use the right tools. Measure risk from the work environment and baseline health in order to track progress. For example, a Health Risk Appraisal instrument that assesses both individual and work-environment health risk factors can help establish baseline workforce health information, direct environmental and individual interventions, and measure progress over time. Optimal assessment of a program's effectiveness is achieved through the use of relevant, validated measurement instruments. 
12. Adjust the program as needed. Successful programs reflect an understanding that the interrelationships between work and health are complex. New workplace programs and policies modify complex systems. Uncertainty is inevitable; consequences of change may be unforeseen. Interventions in one part of a complex system are likely to have predictable and unpredictable effects elsewhere. Programs must be evaluated to detect unanticipated effects and adjusted based on analysis of experience.

13. Make sure the program lasts. Design programs with a long-term outlook to assure sustainability. Shortterm approaches have short-term value. Programs aligned with the core product/values of the enterprise endure. There should be sufficient flexibility to assure responsiveness to changing workforce and market conditions.

14. Ensure confidentiality. Be sure that the program meets regulatory requirements (e.g., HIPAA, State Law, $\mathrm{ADA}$ ) and that the communication to employees is clear on this issue. If workers believe their information is not kept confidential, the program is less likely to succeed.

\section{Program Implementation and Resources}

15. Be willing to start small and scale up. Although the overall program design should be comprehensive, starting with modest targets is often beneficial if they are recognized as first steps in a broader program. For example, target reduction in injury rates or absence. Consider phased implementation of these elements if adoption at one time is not feasible. Use (and evaluate) pilot efforts before scaling up. Be willing to abandon pilot projects that fail.

16. Provide adequate resources. Identify and engage appropriately trained and motivated staff. If you use vendors, make sure they are qualified. Take advantage of credible local and national resources from voluntary and government agencies. Allocate sufficient resources, including staff, space, and time, to achieve the results you seek. Direct and focus resources strategically, reflecting the principles embodied in program design and implementation.

17. Communicate strategically. Effective communication is essential for success. Everyone (workers, their families, supervisors, etc.) with a stake in worker health should know what you are doing and why. The messages and means of delivery should be tailored and targeted to the group or individual and consistently reflect the values and direction of the programs. Communicate early and often, but also have a longterm communication strategy. Provide periodic updates to the organizational leadership and workforce. Maintain program visibility at the highest level of the organization through data-driven reports that allow for a linkage to program resource allocations.

18. Build accountability into program implementation. Accountability reflects leadership commitment to improved programs and outcomes and should cascade through an organization starting at the highest levels of leadership. Reward success.

\section{Program Evaluation}

19. Measure and analyze. Develop objectives and a selective menu of relevant measurements, recognizing that the total value of a program, particularly one designed to abate chronic diseases, may not be determinable in the short run. Integrate data systems across programs and among vendors. Integrated systems simplify the evaluation system and enable both tracking of results and continual program improvement.

20. Learn from experience. Adjust or modify programs based on established milestones and on results you have measured and analyzed. 


\section{Acknowledgements}

We appreciate the contributions of the following individuals who participated in the 2007 workshop leading to the development of this document:

Benjamin Amick, PhD, Scientific Director, Institute for Work \& Health (Canada)

David Anderson, PhD, Vice President, Program Strategy and Development, StayWell

Ron Goetzel, PhD, Vice President, Consulting and Applied Research, Thomson Healthcare

Nico Pronk, PhD, Vice President, Health and Disease Management and Executive Director, Health Behavior Group, HealthPartners

Bonnie Rogers, DrPh, Director, North Carolina, Occupational Safety and Health Education Center and Director, Occupational Health Nursing Program, University of North Carolina at Chapel Hill

Martin Sepulveda, MD, Vice President, Global Occupational Health Services Health Benefits, IBM

Seth Serxner, PhD, Principal, Mercer Health and Benefits

Michael Silverstein, MD, MPH, Clinical Professor, Department of Environmental and Occupational Health Sciences, School of Public Health and Community Medicine, University of Washington

Glorian Sorensen, PhD, MPH, Director, Center for Community-based Research, Dana-Farber Cancer Institute and Professor, Department of Society, Human Development, and Health, Harvard School of Public Health

Laura Welch, MD, Medical Director, Center for Construction Research and Training

\section{This document is in the public domain and may be freely copied or reprinted}

\section{Disclaimer}

Mention of any company or product does not constitute endorsement by the National Institute for Occupational Safety and Health (NIOSH). In addition, citations to Web sites external to NIOSH do not constitute NIOSH endorsement of the sponsoring organizations or their programs or products. Furthermore, NIOSH is not responsible for the content of these Web sites. All web addresses referenced in this document were accessible as of the publication date.

\section{Ordering Information}

To receive niosh documents or more information about occupational safety and health topics, please contact NIOSH:

Telephone: 1-800-cdc-info (1-800-232-4636) /TTY: 1-888-232-6348

email: cdcinfo@cdc.gov or visit the NIOSH Web site at www.cdc.gov/niosh

For a monthly update on news at NIOSH, subscribe to NIOSH eNews by visiting www.cdc.gov/niosh/eNews.

\section{SAFER • HEALTHIER • PEOPLE ${ }^{\mathrm{TM}}$}

DHHS (NIOSH) Publication No. 2010-140 